\title{
Anticholinergic toxicity, polypharmacy, and inappropriate prescribing
}

\author{
Nicole Arseneau (Meds 2018), Kevin Braden (Meds 2017) \\ Faculty Reviewer: Dr Jennie L Wells, BSc, MSc, MD, FRCPC, FACP (Division of Geriatric Medicine)
}

An 84-year-old woman, Doris, is brought to the ER by her daughter just after 2300 on a Tuesday night, complaining of a racing heart beat and palpitations. She is agitated and very uncooperative. Her daughter is worried about how strange Doris is acting. She mentions that the patient had an myocardial infarction (MI) 5 years ago, is diabetic, and takes a number of medications before bed at about 2130 .

Adults over the age of 65 , like Doris, are more likely to visit an emergency department than younger individuals. ${ }^{1,2}$ In addition, older adults tend to have more comorbidities, take more medications (polypharmacy), have higher rates of dementia and other neuropsychiatric disorders, and may present with unconventional and nonspecific symptoms. ${ }^{2-5}$ Unsurprisingly, physicians without specialized geriatric training report feeling less confident when treating older patients in the emergency department compared to younger patients. ${ }^{2}$

Doris is becoming increasingly agitated and confused. On examination, her skin appears erythematous, hot, and dry to the touch. Her oral temperature is $37.8^{\circ} \mathrm{C}$. She has slight bilateral mydriasis. Her respiratory rate is slightly rapid at 20 breaths per minute, her oxygen saturation is $99 \%$, and there is no obstruction of her airway. With some effort, an electrocardiogram is obtained showing sinus tachycardia at 116 beats per minute and her blood pressure is slightly elevated at $140 / 85 \mathrm{mmHg}$. A fingerstick glucose test shows that her blood glucose is $5.8 \mathrm{mmol} / \mathrm{L}$, within normal limits.

\section{ANTICHOLINERGIC TOXICITY}

Doris' attending physician suspects that she is suffering from anticholinergic toxicity due to her classic constellation of symptoms. An anticholinergic toxidrome may present when a medication or poison antagonizes muscarinic receptors throughout the body causing a variety of potentially dangerous effects, often remembered by the mnemonic "hot as a hare (hyperthermia), dry as a bone (anhidrosis), red as a beet (vasodilation), blind as a bat (mydriasis), mad as a hatter (delirium and hallucinations), and full as a flask (urinary retention).” ${ }^{6}$ Anticholinergic effects are more prevalent in the elderly population, in part due to age-related pharmacokinetic and pharmacodynamic changes. ${ }^{7-9}$ Specifically, changes in body composition, including a higher percentage of fat, can increase a drug's volume of distribution and impair elimination of certain drugs, including anticholinergics. Furthermore, a drug's half-life may be significantly prolonged in elderly patients due to liver or kidney impairment. ${ }^{10}$ As such, the American Geriatrics Society recommends that most anticholinergic medications should be avoided in older adults. ${ }^{8}$ Anticholinergic toxicity may result from intentional or unintentional poisoning, overdose, and medical oversight. ${ }^{6,8,12}$ In Doris' case, it is likely that one or more of her medications is causing this acute reaction, though which drug remains unknown.

Doris is moved into an isolated psychiatric care room in the emergency department with her daughter, and her agitation lessens thereafter. Her symptoms do not appear to be life threatening, and have not worsened since her admission. Complete blood count, electrolytes, and arterial blood gases are normal. She continues to be monitored closely.

Treatment of a patient with anticholinergic toxicity varies based on symptoms, time since the exposure, route of administration, patient delirium and aggression, as well as other patient factors. Many cases, such as Doris', can be treated supportively. Activated charcoal can be given to decontaminate the gastric compartment if the suspected agent was given orally. It is most effective if the instigating drug is known to bind to charcoal and was ingested within the last hour. In Doris' case, the drug is unknown and ingestion was most likely over an hour ago. The physician also does not want to interfere with the absorption of her other medications. Physostigmine can be given as an antidote to certain anticholinergic agents in the event of a poisoning; however, it has a number of potential adverse reactions including seizures, bradycardia, heart block, and bronchospasm. ${ }^{13-15}$ Doris' physician is hesitant to start any potentially dangerous treatments when her condition appears to have stabilized.

\section{PRESCRIBING CASCADE}

Doris remains overnight in the emergency department for observation. In the morning, her vitals have partially normalized and she is no longer agitated. She is able to give a complete history, which is significant for an MI 5 years ago, hypertension, seasonal depression, type II diabetes mellitus, and osteoporosis. She is currently taking 13 daily medications for these conditions: propranolol, captopril, low dose ASA, hydrochlorothiazide, metoclopramide, fluoxetine, amitriptyline, rosuvastatin, metformin, naproxen, ranitidine, alendronate, and a calcium/vitamin D supplement. She has also been taking an over the counter (OTC) cold remedy for a persistent cough. She often feels tired during the day and drinks plenty of coffee to stay awake. Recently she has had some trouble falling asleep. On her daughter's advice, she took two diphenhydramine tablets as a sleep aid.

Doris' list of medications is concerning both for its contents as well as its length. Many of her medications are clearly aimed at managing her current conditions; for example, alendronate and her 
calcium/vitamin D supplement are most likely related to her osteoporosis. However, it is possible that some of her medications are simply treating side effects of other medications. This is referred to as a prescribing cascade. Prescribing cascades are common in the elderly population, particularly when the patient is not educated about the potential side effects of a new medication. For example, a persistent cough that Doris is treating with an OTC cold remedy is a common side effect of acetylcholinesterase (ACE) inhibitors such as captopril. Another well known prescribing cascade is the use of metoclopramide to treat nausea associated with any number of drugs. ${ }^{5,16}$ Furthermore, Doris' fatigue may be due to her cardiac medications (most likely propranolol), which may influence her coffee consumption, which in turn could have necessitated her use of diphenhydramine as a sleep aid. The combination of diphenhydramine, an anticholinergic sedating $\mathrm{H}_{1}$ antihistamine, with an OTC cold remedy (likely containing other anticholinergic medications, such as chlorpheniramine and dextromethorphan) is the most likely cause for her hospitalization. ${ }^{8,10,13}$ It is recommended that diphenhydramine use be restricted in all patients of advanced age, except for treatment of severe acute allergic reactions. ${ }^{8}$ While diphenhydramine administration appears to be the precipitating factor for Doris' condition, some of her other medications also have anticholinergic effects, notably amitriptyline and ranitidine. ${ }^{8,11-13}$

Once Doris' symptoms have resolved, she is discharged home with the recommendation that she follow up with her family doctor. She is instructed not to take any more diphenhydramine or OTC cold remedy.

\section{POLYPHARMACY AND INAPPROPRIATE PRESCRIBING}

Doris' case highlights the dangers of polypharmacy, often defined as using more than $\mathbf{5}$ drugs routinely.,12,17 Polypharmacy is increasingly prevalent in the elderly population, with more than $25 \%$ of Canadian adults over the age of 65 taking more than 5 medications daily. ${ }^{9}$ Polypharmacy is believed to be due, at least in part, to more comorbidities in this age range as well as prescribing cascades, as described above. ,9,12,16 $^{2}$ People taking more medications, especially the elderly, are at increased risk for adverse drug reactions, which are a significant cause of hospitalization, morbidity, and mortality. ${ }^{9,10,18} \mathrm{Up}$ to $25 \%$ of older adults report experiencing an adverse drug event within the past year, some requiring hospitalization. ${ }^{918}$ Notably, many older adults are taking OTC, herbal, and dietary preparations in addition to prescription medications, which may contribute to drug interactions and side effects, ${ }^{5}$ as in Doris' case. Nevertheless, polypharmacy itself is not necessarily harmful. Patients may be on several medications, all of which are appropriate for their conditions. ${ }^{11,12}$ What is more concerning in this population is the higher potential for inappropriate prescribing, most likely due to the complexity of medication use. ${ }^{12}$ There are several tools available to physicians to evaluate the appropriateness of a medication in the elderly population, including the Beers Criteria and STOPP Criteria. ${ }^{8,11,12}$ However, even with these tools in place, up to $40 \%$ of nursing home residents in one study were prescribed at least one inappropriate medication..$^{12,17}$

Doris' family physician notes that several of Doris' medications are being used appropriately to treat her conditions, but there are some improvements to be made. The physician suggests replacing captopril, an ACE inhibitor, with an angiotensin II receptor blocker such as losartan. This may relieve Doris' cough while still reducing her blood pressure. In addition, switching from thrice-daily captopril to once-daily losartan may be beneficial as dosing frequency is inversely related to medication compliance. ${ }^{19}$ The physician also suggests that naproxen be replaced with acetaminophen for pain control to reduce the risk of salt retention, hypertension, and GI bleeds. ${ }^{8,11}$ It is recommended that Doris' depression be managed by citalopram instead of her current antidepressants, as amitriptyline is an anticholinergic agent, and fluoxetine is associated with both anorexia and numerous cytochrome P450 interactions. ${ }^{6,8}$ Finally, it is recommended that both ranitidine and metoclopramide be discontinued, as both have anticholinergic properties, and metoclopramide in particular is known to precipitate extrapyramidal and parkinsonian symptoms in the elderly. These reductions and changes in Doris' medications should reduce her overall anticholinergic burden as well as her risk for adverse drug events and interactions.

Polypharmacy, inappropriate prescribing, and adverse drug reactions are an exceedingly common yet preventable source of illness, hospitalization, morbidity, and mortality in the elderly population, yet these undesirable outcomes are also preventable in many cases. $^{9,18}$ It is vitally important for physicians to be mindful of these issues. Physicians should review the indications and contraindications for all medications as well as educate their patients on side effects of both OTC and prescription medications.

\section{REFERENCES}

1. McCusker J, Tousignant P, Borgès Da Silva R, Ciampi A, Lévesque JF, Vadeboncoeur A, Sanche S. Factors predicting patient use of the emergency department: a retrospective cohort study. CMAJ. 2012;184(6):E307-16.

2. Samaras N, Chevalley T, Samaras D, Gold G. Older patients in the emergency department: a review. Ann Emerg Med. 2010;56(3):261-9.

3. Marengoni A, Winblad B, Karp A, Fratiglioni L. Prevalence of chronic diseases and multimorbidity among the elderly population in Sweden. Am J Public Health. 2008;98(7):1198-200.

4. Tinetti ME, Bogardus ST, Agostini J V. Potential pitfalls of disease-specific guidelines for patients with multiple conditions. N Engl J Med. 2004;351(27):2870-4.

5. Qato DM, Alexander GC, Conti RM, Johnson M, Schumm P, Lindau ST. Use of prescription and over-the-counter medications and dietary supplements among older adults in the United States. JAMA. 2008;300(24):2867-78.

6. Gerretsen P, Pollock BG. Drugs with anticholinergic properties: a current perspective on use and safety. Expert Opin Drug Saf. 2011;10(5):751-65.

7. Kachru N, Carnahan RM, Johnson ML, Aparasu RR. Potentially inappropriate anticholinergic medication use in community-dwelling older adults: a national cross-sectional study. Drugs Aging. 2015;DOI:10.100.

8. Fick D, Semla T, Beizer J, et al. American Geriatrics Society updated Beers Criteria for potentially inappropriate medication use in older adults. J Am Geriatr Soc. 2012;60(4):616-31

9. Reason B, Terner M, Moses McKeag A, Tipper B, Webster G. The impact of polypharmacy on the health of Canadian seniors. Fam Pract. 2012;29(4):427-32. 
10. Simons K, Watson W, Martin T, Simons F. Diphenhydramine: pharmacokinetics and pharmacodynamics in elderly adults, young adults, and children. J Clin Pharmacol. 1990;30:665-71.

11. Oliveira MG, Amorim WW, de Jesus SR, Heine JM, Coqueiro HL, Passos LCS. A comparison of the Beers and STOPP criteria for identifying the use of potentially inappropriate medications among elderly patients in primary care. J Eval Clin Pract. 2015;21(2):320-5.

12. Davidoff AJ, Miller GE, Sarpong EM, Yang E, Brandt N, Fick DM. Prevalence of potentially inappropriate medication use in older adults using the 2012 Beers Criteria. J Am Geriatr Soc. 2015;63(3):486-500.

13. Van Hoving DJ, Veale DJH, Müller GF. Clinical review: emergency management of acute poisoning. African J Emerg Med. 2011;1(2):69-78.

14. Suchard JR. Assessing physostigmine's contraindication in cyclic antidepressant ingestions. J Emerg Med. 2003;25(2):185-91.

15. Clinical Practice Guidelines: Management of drug overdose and poisoning. Natl Pharm Adm. 2000;(May):176-263.

16. Kalisch LM, Caughey GE, Roughead EE, Gilbert AL. The prescribing cascade. Aust Prescr. 2011;34(6):162-6.

17. Shelton PS, Fritsch MA, Scott MA. Assessing medication appropriateness in the elderly: a review of available measures. Drugs Aging. 2000;16(6):437-50.

18. Chan M, Nicklason F, Vial JH. Adverse drug events as a cause of hospital admission in the elderly. Intern Med J. 2001;31(4):199-205.

19. Claxton AJ, Cramer J, Pierce C. A systematic review of the associations between dose regimens and medication compliance. Clin Ther. 2001;23(8):1296-310.

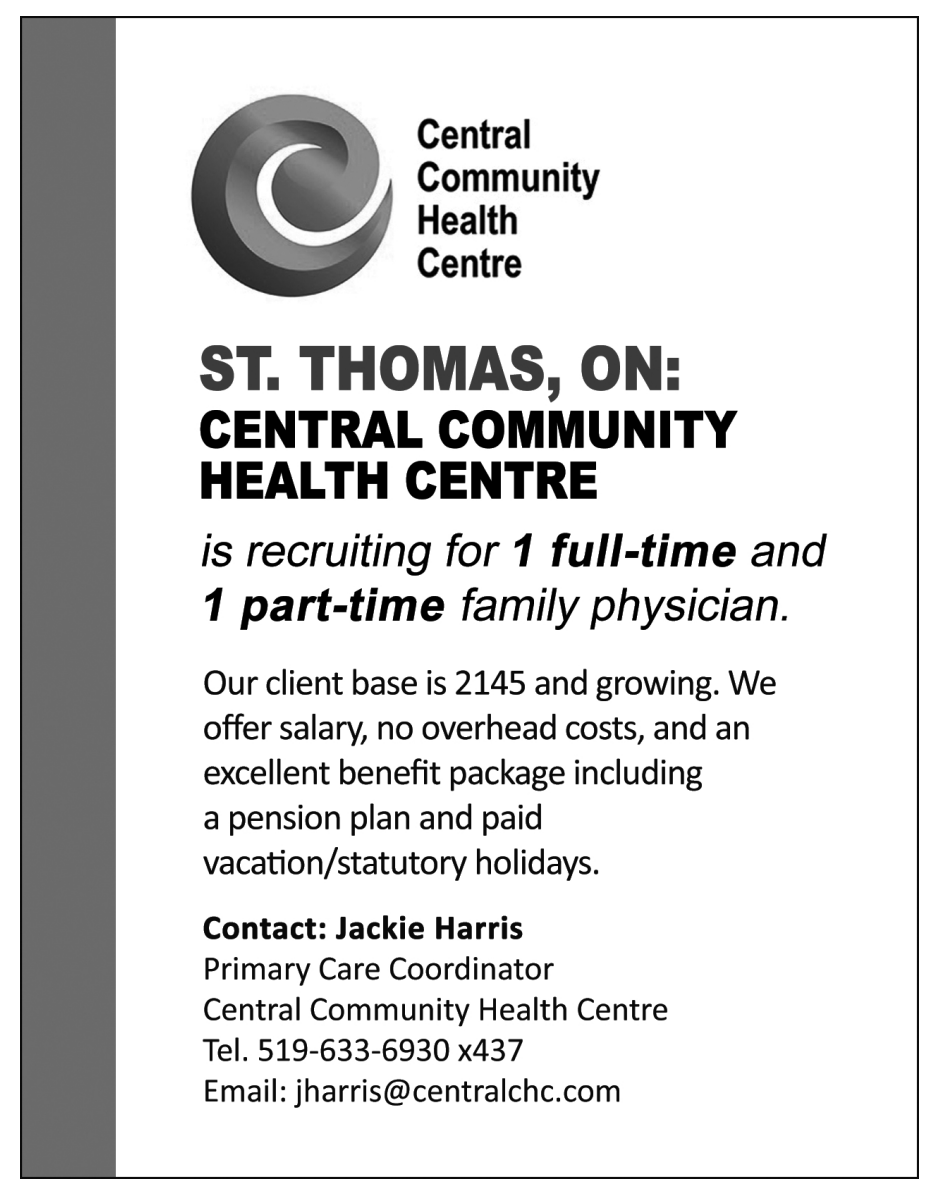

